suggests that neutrophilic airway inflammation may be one such factor although this relationship may be a consequence of coughing rather than causal.

\section{Nadia Yousaf*, William Monteiro*, Sergio Matos", Surinder S. Birring and Ian D. Pavord*}

*Institute of Lung health, Dept of Respiratory Medicine, Glenfield Hospital, Leicester, and 'King's College Hospital, Division of Asthma, Allergy and Lung Biology, London, UK. "Institute of Electronics and Telematics Engineering (IEETA), University of Aveiro, Aveiro, Portugal.

Correspondence: I. Pavord, Institute of Lung health, Dept of Respiratory Medicine, Glenfield Hospital, Groby Road, Leicester, LE3 9QP, UK. E-mail: ian.pavord@uhl-tr.nhs.uk

Support Statement: S. Birring and I.D. Pavord are joint copyright holders of the Leicester Cough Questionnaire.

Statement of Interest: Statements of interest for S. Birring and I. Pavord can be found at www.erj.ersjournals.com/site/misc/ statements.xhtml

\section{REFERENCES}

1 Pavord ID, Chung KF. Management of chronic cough. Lancet 2008; 371: 1375-1384.

2 Morice AH, McGarvey L, Pavord ID. Recommendations for the management of cough in adults. Thorax 2006; 61: 1-24.

3 Birring SS, Fleming T, Matos S, et al. The Leicester Cough Monitor: preliminary validation of an automated cough detection system in chronic cough. Eur Respir J 2008; 31: 1013-1018.

4 Matos S, Birring SS, Pavord ID, et al. Detection of cough signals in continuous audio recordings using hidden Markov models. IEEE Trans Biomed Eng 2006; 53: 1078-1083.

5 Prudon B, Birring SS, Vara DD, et al. Cough and glottic-stop reflex sensitivity in health and disease. Chest 2005; 127: 550-557.

6 Birring SS, Matos S, Patel RB, et al. Cough frequency, cough sensitivity and health status in patients with chronic cough. Respir Med 2006; 100: 1105-1109.

7 Pavord ID, Pizzichini MM, Pizzichini E, et al. The use of induced sputum to investigate airway inflammation. Thorax 1997; 52: 498-501.

8 Birring SS, Prudon B, Carr AJ, et al. Development of a symptom specific health status measure for patients with chronic cough: Leicester Cough Questionnaire (LCQ). Thorax 2003; 58: 339-343.

9 Morice AH, Lowry R, Brown MJ, et al. Angiotensin-converting enzyme and the cough reflex. Lancet 1987; 2: 1116-1118.

DOI: $10.1183 / 09031936.00089312$

\title{
Pleuroparenchymal fibroelastosis as a manifestation of chronic lung rejection?
}

\section{To the Editor:}

Idiopathic pleuroparenchymal fibroelastosis is a peculiar pulmonary fibrosis proposed by FRANKEL et al. [1] in 2003 and is almost the same concept as idiopathic pulmonary upper lobe fibrosis proposed by AMITANI et al. [2]. There are no known causes for fibrosis in idiopathic pleuroparenchymal fibroelastosis. Sometimes, pleuroparenchymal fibroelastosis (PPFE) has underlying diseases or conditions, such as collagen vascular diseases, anti-cancer chemotherapy, irradiation, asbestos exposure and bone-marrow transplantation [3]. Herein, we report the case of a female who received living-donor lung transplantation and died of pulmonary fibrosis, which was pathologically compatible with PPFE in addition to constrictive bronchiolitis, which is a manifestation of chronic lung allograft dysfunction (CLAD) [4].

A 30-yr-old female suffering from idiopathic pulmonary arterial hypertension underwent living-donor lung transplantation surgery and received a right lower lobe from her younger sister and a left lower lobe from her mother in December 2003. 20 months after the lung transplantation she had dyspnoea and a chest radiograph disclosed bilateral ground-glass shadows. 1 month later, right open lung biopsy was performed and a diagnosis of interstitial pneumonia was obtained. Pulse therapy with methylprednisolone slightly improved her condition and prednisolone was administered after the pulse therapy.
However, bilateral interstitial opacities gradually deteriorated (fig. 1) with increased dyspnoea. 49 months after the lung transplantation, her daily life had worsened to almost wholeday bed rest. 18 days prior to her death she noticed fever and general fatigue and was admitted to our hospital (Dept of Respiratory Medicine, Fukuoka University Hospital, Fukuoka, Japan). Pulse therapy using methylprednisolone and antimicrobial and antifungal drugs were administered, without effect. She died 52 months after the lung transplantation. Histological specimens (figs 1a and b) were obtained at autopsy and chest computed tomography (fig. 1c) was obtained 51 months after lung transplantation (1 month before her death). The autopsy revealed a fibrously thickened visceral pleura and marked deposition of elastin just beneath the thickened pleura in both lungs. Alveoli filled with collagen (intra-alveolar fibrosis) were found around the border between the subpleural elastosis and the less involved lung parenchyma. There were foci of constrictive bronchiolitis surrounded by intra-alveolar fibrosis in the lung parenchyma, away from the pleural/subpleural fibrosis and elastosis. The pleural fibrosis, subpleural elastosis and intra-alveolar fibrosis observed in the present case were identical to the pathological features of PPFE described by FRANKEL et al. [1] and were considered as pulmonary fibrosis secondary to lung transplantation.

In 2003, KonEN et al. [5] reported fibrosis of the upper lobes in seven lung transplant recipients. In 2005, PAKHALE et al. [6] also 

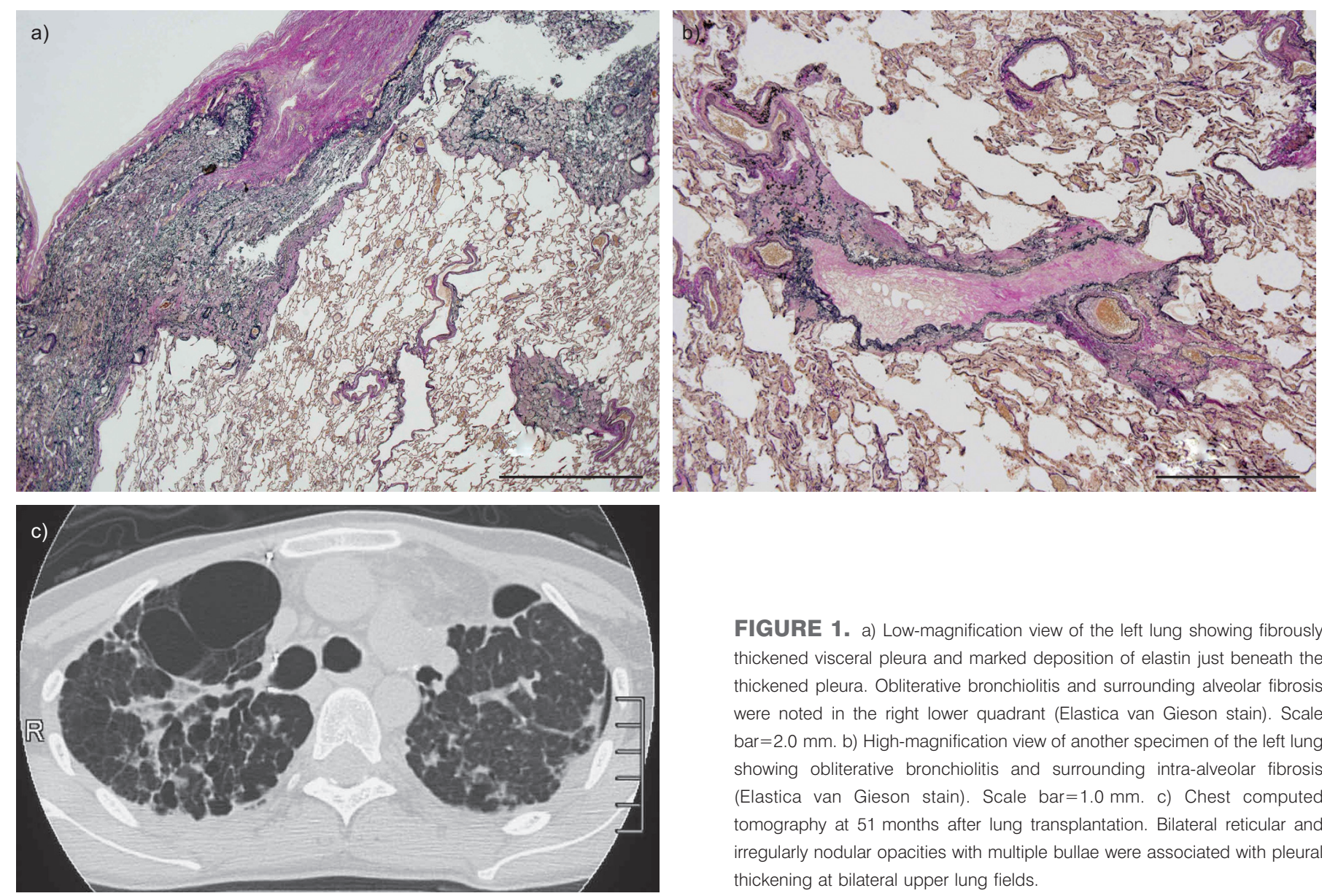

FIGURE 1. a) Low-magnification view of the left lung showing fibrously thickened visceral pleura and marked deposition of elastin just beneath the thickened pleura. Obliterative bronchiolitis and surrounding alveolar fibrosis were noted in the right lower quadrant (Elastica van Gieson stain). Scale bar $=2.0 \mathrm{~mm}$. b) High-magnification view of another specimen of the left lung showing obliterative bronchiolitis and surrounding intra-alveolar fibrosis (Elastica van Gieson stain). Scale bar $=1.0 \mathrm{~mm}$. c) Chest computed tomography at 51 months after lung transplantation. Bilateral reticular and irregularly nodular opacities with multiple bullae were associated with pleural thickening at bilateral upper lung fields.

reported the same concept of the disease in 13 lung transplant recipients. The authors presented imaging characteristics of the fibrosis that started in the upper lobe and expanded to other lobes. Some patients were autopsied, but they did not exhibit any specific histological features for the fibrosis, although some patients had co-existent constrictive bronchiolitis. The authors also suggested the clinical co-existence of bronchiolits obliterans syndrome (BOS) and upper lobe fibrosis in the analysis of the results of respiratory function tests. They concluded that the fibrosis was a novel presentation of chronic allograft dysfunction in lung transplant recipients.

Using autopsy materials, we examined the histological features of the fibrosis in a lung transplant recipient and found that the histology of the fibrosis was identical to that of PPFE. We also identified constrictive bronchiolitis surrounded by peribronchiolar intra-alveolar fibrosis. Intra-alveolar fibrosis and subpleural elastosis are fundamental features of PPFE [7]. These findings raise the possibility that a close relationship exists between upper lobe fibrosis and constrictive bronchiolitis and that the organising process involving the peripheral airway and alveoli without resolution could be the initial step for the disorder, with peripheral airway-dominant lesions resulting in BOS and alveolus-dominant lesions resulting in pulmonary fibrosis.

According to the revised consensus classification of lung allograft rejection by the International Society for Heart and

Lung Transplantation (ISHLT) [8], upper lobe fibrosis, as described by KONEN et al. [5] and PAKHALE et al. [6], is a lateonset complication after lung transplantation that is considered an unhelpful observation because of the lack of specificity and the difficulty in the interpretation of transbronchial biopsy specimens. However, in 2011, SATO et al. [9] proposed the concept of restrictive allograft dysfunction (RAS) as a form of CLAD. CLAD was defined as an irreversible decline in forced expiratory volume in $1 \mathrm{~s}$ (FEV1) to $<80 \%$ of the baseline, and RAS was defined as CLAD with an irreversible decline in total lung capacity (TLC) to $<90 \%$ of the baseline. As FEV1 and TLC in our patient fell to $30 \%$ and $44 \%$, respectively, of the best levels after lung transplantation (baselines) (fig. 2), her ventilatory impairment met the criteria of RAS.

Because RAS was defined functionally, its histological characteristics have not been well established. SATO et al. [9] characterised RAS as various stages of diffuse alveolar damage and extensive fibrosis in the alveolar interstitium with or without constrictive bronchiolitis lesions. In this report, we presented PPFE as a possible pathological phenotype of RAS. Our case might share pathological characteristics with upper lobe fibrosis, which were described by KONEN et al. [5] and PAKHALE et al. [6].

The close relationship between constrictive bronchiolitis and intra-alveolar fibrosis in the present case suggests that PPFE and constrictive bronchiolitis share a common pathway to 

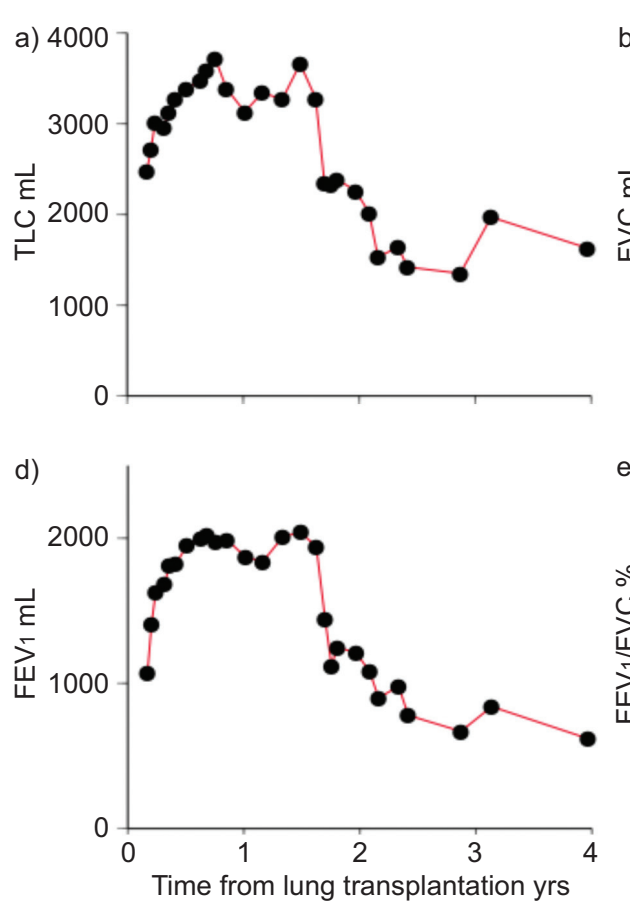

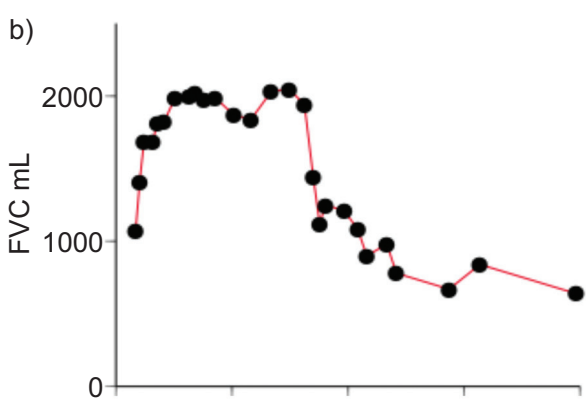

e)

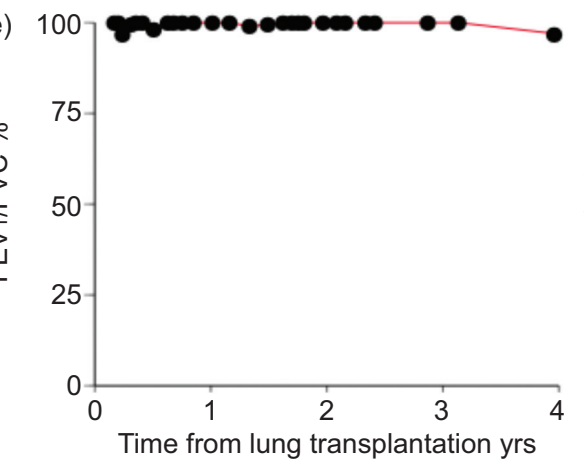

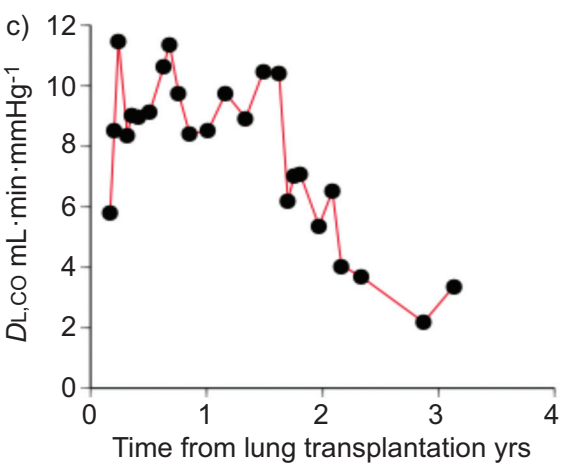

FIGURE 2. Decline of respiratory function after lung transplantation. a) Total lung capacity (TLC), b) forced vital capacity (FVC), c) diffusing capacity of the lung for carbon monoxide $(D L, C O), d)$ forced expiratory volume in $1 \mathrm{~s}$ (FEV1), and e) FEV1/FVC. TLC, FVC, DL,CO and FEV1 at the last measurement fell to $44 \%, 30 \%, 29 \%$ and $30 \%$, respectively, of the best levels after lung transplantation. However, FEV $1 /$ FVC was maintained at almost $100 \%$ during the entire follow-up.
CLAD. PPFE may be a possible pathological phenotype of RAS and a manifestation of CLAD, as is constrictive bronchiolitis.

We showed that lung transplantation is another underlying condition that could induce secondary PPFE. Further studies are needed to elucidate the pathophysiology of transplantationassociated PPFE, which is sometimes a lung manifestation of the chronic graft-versus-host disease observed in bone-marrow transplantation, but is sometimes pathology of CLAD in lung transplantation.

Takako Hirota*, Masaki Fujita*, Takemasa Matsumoto*, Takao Higuchi ${ }^{\#}$, Takeshi Shiraishi", Masato Minami ${ }^{\circledR}$, Meinoshin Okumura ", Kazuki Nabeshima ${ }^{+}$and

\section{Kentaro Watanabe*}

*Dept of Respiratory Medicine, Fukuoka University School of Medicine, "Dept of Thoracic, Endocrine and Paediatric Surgery, Fukuoka University School of Medicine, ${ }^{+}$Dept of Pathology, Fukuoka University School of Medicine, Fukuoka, and "Dept of General Thoracic Surgery, Osaka University Graduate School of Medicine, Osaka, Japan.

Correspondence: K. Watanabe, Dept of Respiratory Medicine, Fukuoka University School of Medicine, Fukuoka 810-0180, Japan. E-mail: watanabe@fukuoka-u.ac.jp

Support Statement: This work was partly supported by a grant to the Diffuse Lung Diseases Research Group from the Ministry of Health, Labour and Welfare, Japan.
Statement of Interest: None declared.

\section{REFERENCES}

1 Frankel SK, Cool CD, Lynch DA, et al. Idiopathic pleuroparenchymal fibroelastosis: description of a novel clinicopathological entity. Chest 2003; 126: 2007-2013.

2 Amitani R, Niimi A, Kuse F. [Idiopathic pulmonary upper lobe fibrosis]. Kokyu 1992; 11: 693-699.

3 von der Thusen JH, Hansell DM, Veys PA, et al. Pleuroparenchymal fibroelastosis in patients with pulmonary disease secondary to bone marrow transplantation. Modern Pathol 2011; 24: 1633-1639.

4 Woodrow JP, Shlobin OA, Barnett SD, et al. Comparison of bronchiolitis obliterans syndrome to other forms of chronic lung allograft dysfunction after lung transplantation. J Heart Lung Transplant 2010; 29: 1159-1164.

5 Konen E, Weisbrod GL, Pakhale S, et al. Fibrosis of the upper lobes: a newly identified late-onset complication after lung transplantation? AJR Am J Roentogenol 2003; 181: 1539-1543.

6 Pakhale SS, Hadjiliadis D, Howell DN, et al. Upper lobe fibrosis: a novel manifestation of chronic allograft dysfunction in lung transplantation. J Heart Lung Transplant 2005; 24: 1260-1268.

7 Reddy TL, Tominaga M, Hansell DM, et al. Pleuroparenchymal fibroelastosis: a spectrum of histopathological and imaging phenotypes. Eur Respir J 2012; 40: 377-385.

8 Stewart S, Fishbein MC, Snell GI, et al. Revision of the 1996 working formulation for the standardization of nomenclature in the diagnosis of lung rejection. J Heart Lung Transplant 2007; 26: 1229-1242.

9 Sato M, Waddell TK, Wagnetz U, et al. Restrictive allograft syndrome (RAS): a novel forms of chronic lung allograft dysfunction. J Heart Lung Transplant 2011; 30: 735-742.

DOI: $10.1183 / 09031936.00103912$ 